\title{
BMI open Long-term sustained remission in a cohort study of patients with rheumatoid arthritis: choice of remission criteria
}

\author{
Björn Svensson, ${ }^{1}$ Maria L E Andersson, ${ }^{2}$ Sidona-Valentina Bala, ${ }^{3}$ \\ Kristina Forslind, ${ }^{1,3}$ Ingiäld Hafström, ${ }^{4}$ on behalf of the BARFOT study group
}

To cite: Svensson B, Andersson MLE, Bala S-V et al. Long-term sustained remission in a cohort study of patients with rheumatoid arthritis: choice of remission criteria. BMJ Open 2013;3: e003554. doi:10.1136/ bmjopen-2013-003554

- Prepublication history for this paper is available online. To view these files please visit the journal online (http://dx.doi.org/10.1136/ bmjopen-2013-003554).

Received 5 July 2013 Accepted 19 July 2013

\footnotetext{
${ }^{1}$ Department of Clinical Sciences, Section of Rheumatology, Lund University, Lund, Sweden ${ }^{2} \mathrm{R}$ and $\mathrm{D}$ Center, Spenshult Hospital, Oskarström, Sweden

${ }^{3}$ Department of Internal Medicine, Section of Rheumatology, Helsingborgs Lasarett, Helsingborg, Sweden

${ }^{4}$ Department of Rheumatology, Karolinska Institutet at Karolinska University Hospital, Huddinge, Sweden

Correspondence to Dr Björn Svensson; bjosve@telia.com
}

\begin{abstract}
Objectives: Remission is a widely accepted goal for treatment of rheumatoid arthritis (RA) but has to be sustained to arrest joint damage and disability. However, appropriate criteria for the assessment of sustained remission in long-term studies are not established. Therefore, we have compared the disease activity score calculated on 28 joints (DAS28) remission criterion, the Simplified Disease Activity Index less than 3.3 remission criterion (SDAI $\mathrm{Cr}$ ) and the new Booleanbased set of criteria (Boolean $\mathrm{Cr}$ ), and assessed the association of these criteria with radiographic and functional outcome.
\end{abstract}

Design: Prospective, long-term observational study of patients with early RA.

Setting: Secondary level of care; six participating centres from southern Sweden; both urban and rural populations.

Participants: 698 patients were consecutively included in the study and 527 remained at the 8-year follow-up visit. Almost all patients were Caucasians, of which $64 \%$ were women. To be included, a patient, 18 years or older, had to fulfil the 1987 American College of Rheumatology criteria for RA and have a disease duration of no more than 1 year.

Results: Sustained remission was most common by the DAS28 $\mathrm{Cr}(14 \%)$, while $3 \%$ met the Boolean $\mathrm{Cr}$ and $5 \%$ the SDAI $\mathrm{Cr}$, the latter figures increasing to $9 \%$ and $8 \%$, respectively, when the patient's global assessment was excluded. Radiographic joint damage was common but least pronounced in patients in sustained remission by all criteria. Sustained remission was associated with rapid and lasting improvement in function assessed by the Health Assessment questionnaire, irrespective of criteria.

Conclusions: The DAS28 $\mathrm{Cr}$ acquired more patients in sustained remission compared with the other criteria. In spite of that, radiographic damage and disability were not worse than that seen by other criteria and the patients' perspective was preserved. The DAS28 Cr may therefore still be used in long-term observational studies until more accurate criteria are available.

\section{ARTICLE SUMMARY}

Article focus

- To assess the prevalence of sustained remission in early rheumatoid arthritis (RA) during the first 8 years after inclusion in the study.

- To study the feasibility in long-term studies of the most frequently used remission criteria, the disease activity score calculated on 28 joints (DAS28) remission criterion and the new American College of Rheumatology/EU criteria.

- To assess the association of these criteria with radiographic and functional outcome.

Key messages

- Sustained remission was infrequent by all criteria used but most frequent by the DAS28 criterion.

- Patients in sustained remission by the DAS28 criterion did not have worse function or more evidence of joint destruction compared with the more stringent criteria.

- The DAS28 criterion may be used in long-term studies until more accurate criteria become available.

Strengths and limitations of this study

- Data are derived from a cohort of patients with early RA followed in a structured way for up to 8 years.

- In addition to clinical data, radiographs are available for the evaluation of the progression of joint damage.

- One hundred and seventy-one patients were lost to follow-up after 8 years. For 41 of these patients the reason is unknown.

- Flares of disease activity may have been missed due to the scarcity of follow-up visits during the 8-year follow-up.

\section{INTRODUCTION}

The principal aim in the treatment of rheumatoid arthritis (RA) is to suppress the inflammatory process (the disease activity) 
and achieve remission. Remission may be defined as a state with no or little disease activity. However, remission must be sustained in order to eliminate or arrest joint damage. ${ }^{1}$

Several sets of criteria for remission have been proposed and applied in a number of studies of RA. The original American Rheumatism Association (ARA) remission criteria are infrequently used today since all components of the criteria are not included in the current core set of variables. ${ }^{2}$ A Disease Activity Score (DAS) less than 1.6 was found to correspond well to the ARA remission criteria and was proposed as a remission criterion. ${ }^{3}{ }^{4}$ Later, the DAS remission criterion was modified by a 28 joint count to the DAS28 less than 2.6 criterion (DAS28 Cr), which has been widely used. Since then, more stringent criteria have been developed, for example, the Simplified Disease Activity Index less than 3.3 remission criterion (SDAI Cr) ${ }^{5}$ Recently, the American College of Rheumatology (ACR) and the European League Against Rheumatism (EULAR), in collaboration, proposed that remission in RA may be defined either according to the SDAI Cr or to the new Boolean-based set of criteria (Boolean Cr). ${ }^{6}$ The Boolean $\mathrm{Cr}$ have been shown to perform well in clinical trials, but their utility in long-term observational studies remains to be demonstrated. ${ }^{6}$ The most frequently used criterion, the DAS28 Cr, has been questioned since patients may be in remission by this criterion in spite of several swollen and tender joints.

On the basis of these considerations, long-term sustained remission by different criteria has been studied in the Better AntiRheumatic PharmacOTherapy (BARFOT) observational study of patients with early RA and related to disability and radiographic joint damage. ${ }^{7}$

\section{PATIENTS AND METHODS}

\section{Patients}

In all, 698 patients with RA were consecutively included in the BARFOT observational study ${ }^{7}$ from September 1995 to September 1999 and 527 of these have completed 8 years. In total, 171 patients were lost to follow-up; of these, 119 had died, 9 had moved, 2 rejected further follow-up visits and in 41 cases the cause was unknown.

The group of patients lost to follow-up were older (mean age 67 vs 54 years, $\mathrm{p}=0.001$ ), had a higher score on the Health Assessment questionnaire (HAQ; mean 1.09 vs $0.97, \mathrm{p}=0.043$ ) and were somewhat less frequently positive for antibodies to citrullinated proteins (ACPA; $49 \%$ vs $58 \%, \mathrm{p}=0.044)$.

All patients had to fulfil the ACR criteria for classification of RA and have a disease duration of 12 months or less. The patients were checked by a structured protocol at baseline and after 6 months and 1, 2, 5 and 8 years. A few patients had been treated before inclusion with disease modifying drugs (DMARDs) or glucocorticoids (GCs) but treatment was in most patients initiated at inclusion (baseline). The patients were treated by the rheumatologists' preferences.

\section{METHODS}

\section{Clinical assessments}

Disease activity was measured by the number of swollen and tender joints calculated on 28 joints, patient global assessment of disease activity (PatGA) on a visual analogue scale (VAS) of $10 \mathrm{~cm}$, and the physician's global assessment of the disease activity (PhGA) measured on a five-stage Likert scale, which was transformed to a VAS of $10 \mathrm{~cm}$.

Erythrocyte sedimentation rate (ESR) was measured by the Westergren method and $\mathrm{C}$ reactive protein (CRP; $\mathrm{mg} / \mathrm{dL}$ ) by a standard non-high sensitive method. The pain experienced by the patient was assessed on a VAS. Disability was assessed by the Swedish version of the Stanford HAQ. ${ }^{8}$ ACPA were detected using the ELISA CCP2 test (anti-CCP, Euro-Diagnostica, Malmö, Sweden).

Remission was defined according to the following criteria:

- The DAS28 remission criterion (DAS28 Cr): DAS calculated on 28 joints (DAS28) is a combined index which includes the number of swollen and tender joints, the patient's global assessment and ESR. ${ }^{9}$ Remission is defined as DAS28<2.6. ${ }^{10}$ This was the criterion used in clinical practice when deciding on treatment in the present population.

- The recently proposed Boolean-based ACR/EULAR remission criteria (Boolean $\mathrm{Cr}$ ): The tender joint and swollen joint counts $\leq 1$, PatGA $\leq 1 \mathrm{~cm}$ on a VAS of $10 \mathrm{~cm}$ and CRP $\leq 1 \mathrm{mg} / \mathrm{dL}^{6}{ }^{10}$

- The SDAI remission criterion (SDAI Cr): The sum of the number of swollen and tender joints, CRP (mg/ dL), PatGA and PhGA $\leq 3.3$.

- 'DAS28-3 Cr', 'Boolean-3 Cr' and 'SDAI-4 Cr': The only difference from the original criteria is that VAS PatGA has been excluded.

Sustained remission was defined as remission at all four follow-up visits at 1, 2, 5 and 8 years, never remission as absence of remission at all visits, while intermittent remission was defined as remission at one, two or three of these four follow-up visits.

\section{Radiographic assessment}

The posterior-anterior radiographs of the hands and feet were obtained at study entry in 630 patients, at 1 year in 594 patients, at 2 years in 613 patients, at 5 years in 560 patients and at 8 years in 468 patients. Patients not having any radiographs did not differ significantly in baseline characteristics from patients with radiographs (data not shown).

Radiographic joint damage was assessed according to the van der Heijde modification of the Sharp score. ${ }^{11}$ The Sharp van der Heijde total scores (SHS) are presented (range 0-448). The films were read by one of two experienced readers. Double readings of a fraction of 
Table 1 Demographic and clinical characteristics of the 527 RA patients

\begin{tabular}{|c|c|c|c|}
\hline & & Per cent & Mean (SD) \\
\hline Inclusion age, years & & & 57 (15) \\
\hline Disease duration, months & & & $6.2(3.2)$ \\
\hline Gender & Women & 64 & \\
\hline Ever smokers & & 55 & \\
\hline Anti-CCP & Positive & 56 & \\
\hline Rheumatoid factor & Positive & 60 & \\
\hline VAS pain $(0-10 \mathrm{~cm})$ & & & $4.5(2.4)$ \\
\hline DAS28 & & & $5.23(1.2)$ \\
\hline CRP (mg/L) & & & $35(37)$ \\
\hline $\begin{array}{l}\text { Tender joint count } \\
\text { (28 joints) }\end{array}$ & & & $8(6)$ \\
\hline $\begin{array}{l}\text { Swollen joint count } \\
\text { ( } 28 \text { joints) }\end{array}$ & & & $11(6)$ \\
\hline VAS PatGA $(0-10 \mathrm{~cm})$ & & & $4.4(2.5)$ \\
\hline VAS PhGA $(0-10 \mathrm{~cm})$ & & & $4.8(1.7)$ \\
\hline $\mathrm{HAQ}(0-3)$ & & & $1.0(0.65)$ \\
\hline SHS (median (IQR)) & & & $1(0-4.5)$ \\
\hline Glucocorticoids & Yes & 42 & \\
\hline DMARDS & No & 13 & \\
\hline MTX & & 40 & \\
\hline SAL & & 34 & \\
\hline Other & & 12 & \\
\hline Combination & & 1 & \\
\hline Biologics & & 0 & \\
\hline \multicolumn{4}{|c|}{$\begin{array}{l}\text { Anti-CCP, antibodies to citrullinated peptides; CRP, C reactive } \\
\text { protein; DAS28, disease activity score calculated on } 28 \text { joints; } \\
\text { HAQ, Health Assessment Questionnaire; PatGA, patients global } \\
\text { assessment; PhGA, physician's global assessment; MTX, } \\
\text { methotrexate; RA, rheumatoid arthritis; SHS, Sharp van der Heijde } \\
\text { total Score; SAL, sulfasalazine; VAS, visual analogue scale. }\end{array}$} \\
\hline
\end{tabular}

films showed good agreement between the two readers (data not shown).

Radiographic progression was defined as a change in SHS of more than 1 unit per year, based on the assumption that a change of 1 unit/year is the lowest value of minor radiographic change. ${ }^{12} 13$

\section{Statistical analysis}

Statistical analyses were performed using SPSS V. 21.0 statistical software. To test the differences between groups, the Mann-Whitney U test and the Kruskal-Wallis test were used for continuous variables, and the $\chi^{2}$ test for proportions. Pearson's correlation test was used to assess the relations between two continuous variables.
Positive likelihood ratios for the ability of sustained remission to predict favourable radiographic outcome were calculated by the formula sensitivity/(1-specificity). All significance tests were two tailed and conducted at the 0.05 significance level.

\section{RESULTS}

\section{Demographic and clinical baseline characteristics}

At baseline, the mean age of the patients was 57 years and $64 \%$ were women. The mean DAS28 was 5.23 , the mean HAQ was 1.0 and the median SHS was 1 . Forty-two per cent of the patients were started on GC treatment and $87 \%$ on non-biological DMARDs (table 1).

\section{Proportion of patients in remission at the follow-up visits}

The frequencies of remission increased from 6 months and onwards by all criteria used. Table 2 shows that the remission rates from 1 to 8 years were most frequent by the DAS28 based criteria, irrespective of whether PatGA was excluded or not. Remissions by the Boolean $\mathrm{Cr}$ and the SDAI Cr were less frequent but the frequencies increased when PatGA was excluded from the criteria.

\section{Number of patients in remission at one, two, three or all} of the four follow-up visits

Table 3 shows the number of patients in remission at the follow-up visits between 1 and 8 years. Sustained remission was achieved by $14 \%$ by the DAS28 Cr, $3 \%$ by the Boolean $\mathrm{Cr}$ and $5 \%$ by the SDAI Cr. Thirty-five per cent of the patients had no episode of remission at all (never remission) by the DAS28 Cr, $62 \%$ by the Boolean $\mathrm{Cr}$ and $58 \%$ by the SDAI Cr. The remaining patients had one, two or three episodes of remission (intermittent remission). PatGA is included in all these remission criteria. With low cut-off values for PatGA, remission may be difficult to achieve. When PatGA was excluded from the criteria, the rates of sustained remission increased to $9 \%$ by the Boolean-3 Cr and to $8 \%$ by the SDAI- 4 Cr but decreased to $12 \%$ by the DAS28-3 Cr.

\section{Influence of the number of tender and swollen joints on sustained remission}

The DAS28 formula allows the classification of a patient as in remission even in the presence of several swollen or tender joints. To investigate whether this might also be the case in patients in sustained remission in this

Table 2 Remission rates at the follow-up visits (number (\%)) at 1,2, 5 and 8 years according to the criteria used

\begin{tabular}{lccrr}
\hline & 1 year & 2 years & 5 years & 8 years \\
\hline DAS28 Cr & $175(36.2)$ & $189(39.1)$ & $190(39.3)$ & $202(41.8)$ \\
DAS28-3 Cr & $166(33.1)$ & $188(37.5)$ & $178(35.5)$ & $198(39.5)$ \\
Boolean Cr & $88(17.3)$ & $93(18.3)$ & $84(16.5)$ & $97(19.1)$ \\
Boolean-3 Cr & $136(27.2)$ & $145(29)$ & $158(31.6)$ & $189(37.8)$ \\
SDAI Cr & $124(19)$ & $153(22.6)$ & $145(24.4)$ & $115(23.6)$ \\
SDAl-4 Cr & $173(26.3)$ & $195(28.7)$ & $210(34.9)$ & $184(37.4)$ \\
\hline DAS28, disease activity score calculated on 28 joints; SDAI Cr, Simplified Disease Activity Index less than 3.3 remission criterion.
\end{tabular}


Table 3 Patients in sustained, intermittent or never remission by the different criteria

\begin{tabular}{|c|c|c|c|c|c|}
\hline & \multicolumn{5}{|c|}{ Number (\%) of patients in remission at all four, three, two, one or no visits } \\
\hline & \multirow{2}{*}{$\begin{array}{l}\text { Sustained remission } \\
\text { All four visits }\end{array}$} & \multicolumn{3}{|c|}{ Intermittent remission } & \multirow{2}{*}{$\begin{array}{l}\text { Never remission } \\
\text { No visit }\end{array}$} \\
\hline & & Three & Two & One & \\
\hline DAS28 Cr & $69(14)$ & $76(16)$ & $81(17)$ & 90 (19) & $167(35)$ \\
\hline DAS28-3 Cr & $60(12)$ & $75(15)$ & $88(17)$ & $89(18)$ & $189(38)$ \\
\hline Boolean $\mathrm{Cr}$ & $14(3)$ & $32(6)$ & $62(11)$ & $86(17)$ & $315(62)$ \\
\hline Boolean-3 Cr & $45(9)$ & $51(10)$ & $90(18)$ & $115(23)$ & 199 (40) \\
\hline SDAI Cr & $22(5)$ & $34(8)$ & 67 (16) & 56 (13) & $245(58)$ \\
\hline SDAI-4 Cr & $39(8)$ & $61(13)$ & $104(22)$ & 87 (18) & $186(39)$ \\
\hline
\end{tabular}

study, the number of tender and swollen joints, which were components of DAS28 in these patients, was counted. Table 4 shows that more than one or two swollen or tender joints were infrequent.

\section{Drug treatment}

At baseline, 1 and 2 years, information on GC and DMARD treatment was available in all or almost all patients while no such information was available at 5 years in $11 \%$ and $13 \%$ and at 8 years in $27 \%$ and $28 \%$, respectively. At baseline, there were no statistically significant differences between remission groups in the frequency and kind of DMARD treatment. At the follow-up visit at 8 years, significantly more patients in the sustained remission groups (48-64\%) had stopped DMARDs than was the case in the intermittent (26-35\%) and never remission groups (19-23\%). The differences were statistically significant, irrespective of the criteria used, $\mathrm{p}<0.002$ or less. Only $0-5 \%$ of the patients in the sustained remission groups were treated with biologics versus $9-15 \%$ and $16-18 \%$ of the patients in the intermittent and never remission groups, respectively. Post hoc analyses showed that this was significant when the DAS28-based criteria and the Boolean-3 $\mathrm{Cr}$ were used.

At baseline, $42 \%$ of the patients in all remission groups were started on GCs. After 8 years, fewer patients in sustained remission were treated with GCs $(0-5 \%)$ than in the intermittent (16-20\%) and the never remission groups (24-28\%). The differences were statistically significant, irrespective of the criteria used ( $p<0.03$ or less).

\section{Radiographic joint damage in the sustained remission groups}

Radiographic joint damage as assessed by SHS increased significantly from years 1 to 8 in all remission groups, least in the sustained remission groups and most in the groups with no remissions at all (figure 1A-F). In the sustained remission groups, the mean (SD) increases in SHS between baseline and 8 years were similar: $7.4(8.6)$ by the DAS28 Cr, $7.3(9.3)$ by the DAS28-3 Cr, 7.2 (9.2) by the Boolean $\mathrm{Cr} ; 7.8$ (8.4) by the Boolean-3 Cr, 8.0 (15) by the SDAI Cr and 8.7 (13.1) by the SDAI-4 Cr.

Radiographic progression, defined as a change of more than 1 unit/year between baseline and 8 years, occurred in $38 \%$ of the patients in sustained remission by the DAS28 Cr. The corresponding figures for patients in sustained remission by the DAS28-3 Cr, Boolean Cr, Boolean-3 Cr, SDAI Cr and SDAI-4 Cr were similar: 37\%, $31 \%, 45 \%, 26 \%$ and $40 \%$, respectively.

\section{Performance of the criteria}

Table 5 displays the performance of the criteria. There was a general tendency for sustained remission to be associated with absence of radiographic progression. The association varied somewhat between criteria; the sensitivity was low and the likelihood ratios were small and not statistically significant for the Boolean-based and SDAI-4 criteria.

\section{Disability in the sustained remission groups}

Disability assessed by HAQ decreased after 6 months in all remission groups by all criteria used, being most pronounced in the sustained remission groups and least in the never remission groups(figure 2A-F). Thereafter, HAQ remained more or less on that lower level during the rest of the study. At baseline, the sustained remission groups had lower HAQ and remained on an HAQ score of about 0.2 or less during the rest of the study while the groups of patients who never achieved remission remained on an HAQ score of 0.8 or higher.

Table 4 Number of patients in remission by DAS28 Cr with more than one tender or swollen joint

\begin{tabular}{llllll}
\hline & 2 Joints & 3 Joints & 4 Joints & 5 Joints & 6 Joints \\
\hline Tender joint count $>1$ & 12 & 1 & 2 & 1 & 0 \\
Swollen joint count $>1$ & 22 & 7 & 6 & 2 & 1 \\
\hline Results from 276 assessments of DAS28 in patients with sustained remission. \\
DAS28, disease activity score calculated on 28 joints.
\end{tabular}




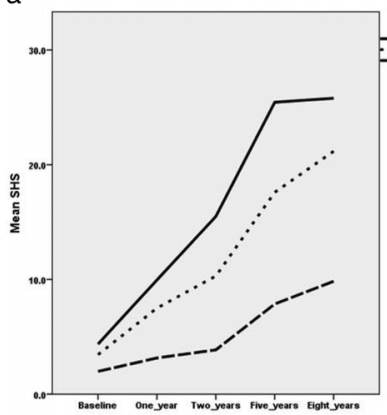

d

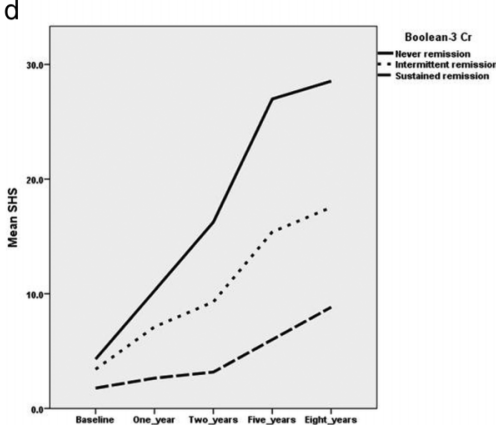

b

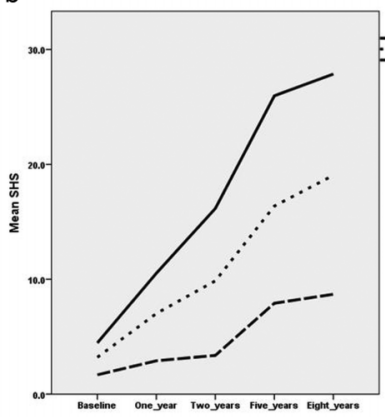

e
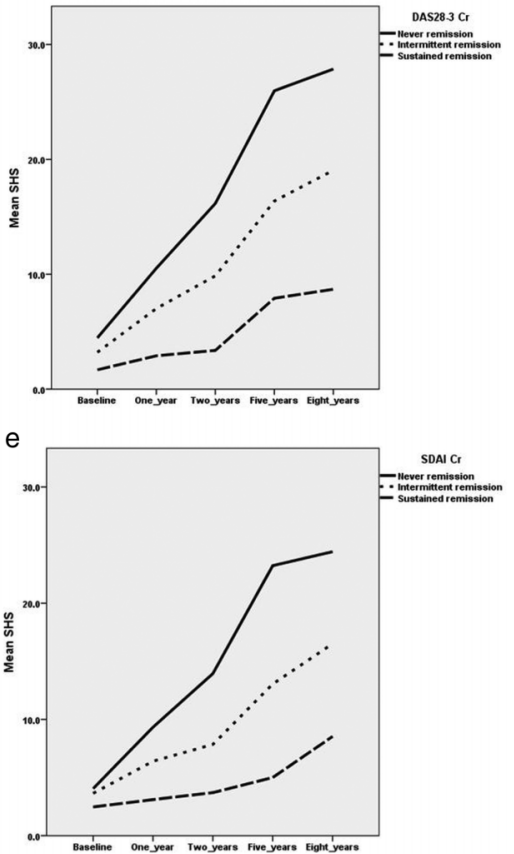
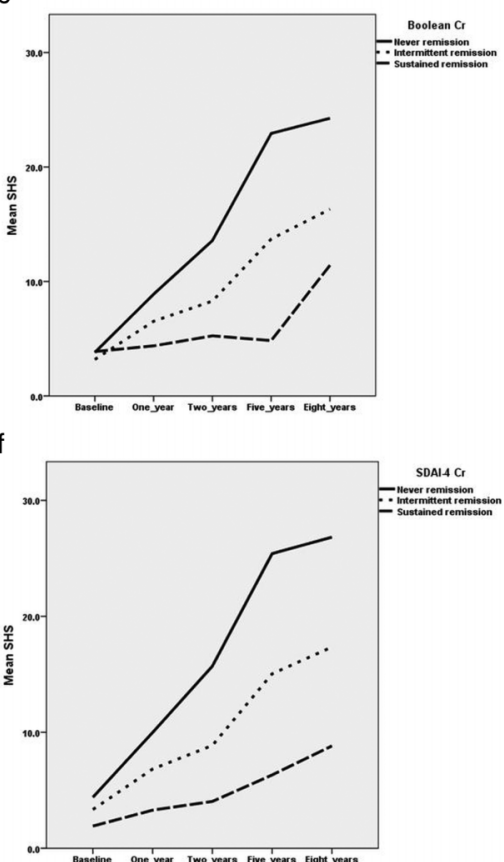

Figure 1 (A-F) The mean Sharp van der Heijde total Score from baseline to 1, 2, 5 and 8 years in patients in sustained remission, intermittent remission or never in remission by DAS28 $\mathrm{Cr}(\mathrm{A})$, DAS28-3 $\mathrm{Cr}(\mathrm{B})$, Boolean $\mathrm{Cr}(\mathrm{C})$, Boolean-3 $\mathrm{Cr}(\mathrm{D})$, SDAI $\mathrm{Cr}(\mathrm{E})$, SDAI-4 $\mathrm{Cr}(\mathrm{F})$. At baseline, there was a statistically significant difference overall only for DAS28-3 $\mathrm{Cr}, \mathrm{p}=0.002$ (Kruskal-Wallis test). At the follow-up visits, there was a statistically significant difference overall with $p<0.001$ between remission groups except for the Boolean $\mathrm{Cr}$ at 1 year, $\mathrm{p}=0.046$, at 2 years, $\mathrm{p}=0.002$ and at 8 years, $\mathrm{p}=0.013$ and for the SDAI $\mathrm{Cr}$ at 1 year, $\mathrm{p}=0.011$ and at 8 years, $\mathrm{p}=0.008$.

Improvement in function was defined as a change in HAQ from baseline to 8 years of 0.22 or more. ${ }^{14}$ Irrespective of the criteria, sustained remission was associated with improvement. By the DAS28 Cr, $73 \%$ of the patients in sustained remission improved after 8 years versus $62 \%$ of the patients with intermittent remission and $40 \%$ of the patients who never achieved remission. The corresponding figures for the DAS28-3 Cr, the Boolean Cr, the Boolean Cr-3, the SDAI Cr and the SDAI- $4 \mathrm{Cr}$ were $80 \%, 58 \%$ and $45 \%$; $79 \%, 57 \%$ and $48 \%$; $73 \%, 61 \%$ and $43 \% ; 73 \%, 67 \%$ and $45 \% ; 69 \%, 63 \%$ and
$41 \%$. All comparisons were statistically significant, overall $\mathrm{p}=0.001$.

\section{Sustained remission and gender}

Irrespective of the criteria used, the proportion of men in sustained remission was higher than that of women. Thus, by the DAS28 Cr, $10 \%$ of the women versus $23 \%$ of the men achieved sustained remission, $\mathrm{p}=0.001$, and by the DAS28-3 Cr 9\% vs $19 \%, p=0.001$. The respective rates were by the Boolean $\mathrm{Cr} 2 \%$ vs $5 \%, \mathrm{p}=0.008$; by the

Table 5 The performance of the different criteria in patients in sustained remission

\begin{tabular}{|c|c|c|c|c|c|c|c|c|}
\hline & \multicolumn{2}{|c|}{ Non-progressors* } & \multirow[b]{2}{*}{ Sensitivity } & \multirow[b]{2}{*}{ Specificity } & \multirow[b]{2}{*}{ PPV } & \multirow[b]{2}{*}{ NPV } & \multirow[b]{2}{*}{ p Value } & \multirow[b]{2}{*}{$\begin{array}{l}\text { Likelihood ratio } \\
(95 \% \mathrm{Cl})\end{array}$} \\
\hline & $\begin{array}{l}\text { Percentage } \\
\text { in sustained } \\
\text { remission }\end{array}$ & $\begin{array}{l}\text { Percentage not } \\
\text { in sustained } \\
\text { remission }\end{array}$ & & & & & & \\
\hline DAS28 Cr & 62 & 45 & 0.19 & 0.90 & 0.62 & 0.55 & 0.015 & $1.82(1.11$ to 2.97$)$ \\
\hline DAS28-3 Cr & 63 & 45 & 0.16 & 0.91 & 0.63 & 0.53 & 0.022 & 1.77 (1.04 to 3.02$)$ \\
\hline Boolean Cr & 62 & 47 & 0.05 & 0.98 & 0.69 & 0.53 & 0.11 & 2.47 (0.77 to 7.91$)$ \\
\hline Boolean-3 Cr & 55 & 47 & 0.12 & 0.91 & 0.55 & 0.53 & 0.32 & $1.33(0.75$ to 2.38$)$ \\
\hline SDAI Cr & 74 & 47 & 0.08 & 0.97 & 0.74 & 0.53 & 0.025 & 2.96 (1.09 to 8.05$)$ \\
\hline SDAl-4 Cr & 60 & 47 & 0.11 & 0.93 & 0.60 & 0.53 & 0.14 & 1.62 (0.8 to 3.09$)$ \\
\hline
\end{tabular}


a

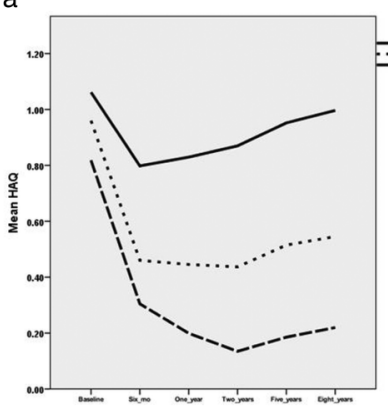

d

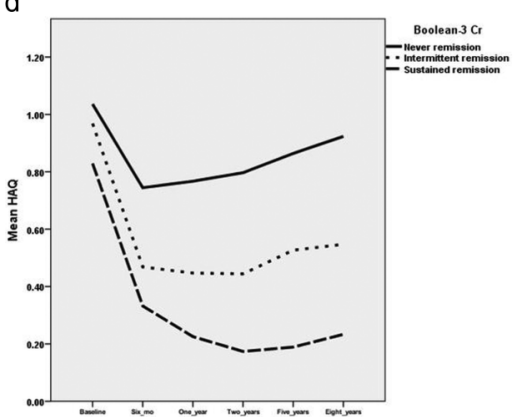

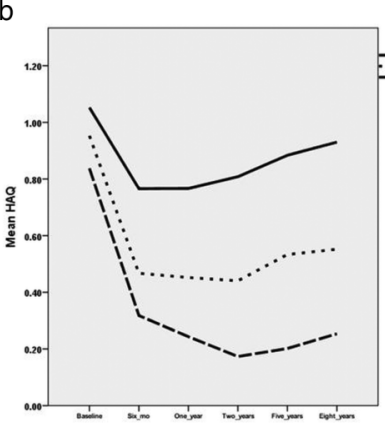

e
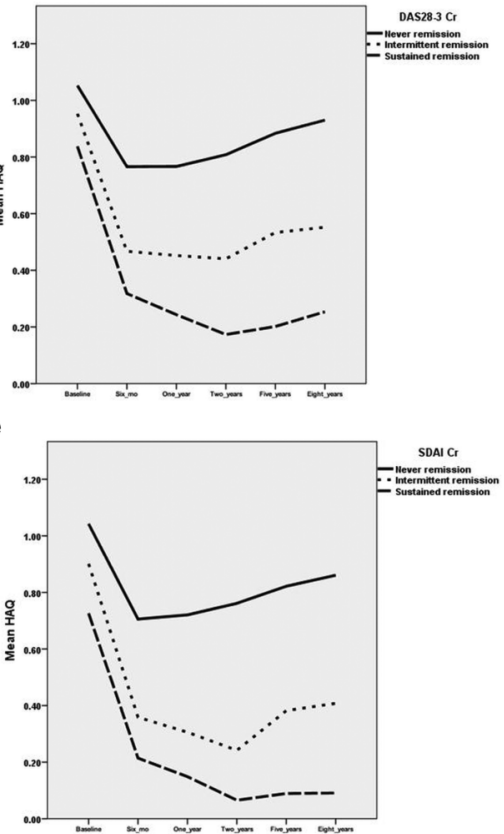

$f$

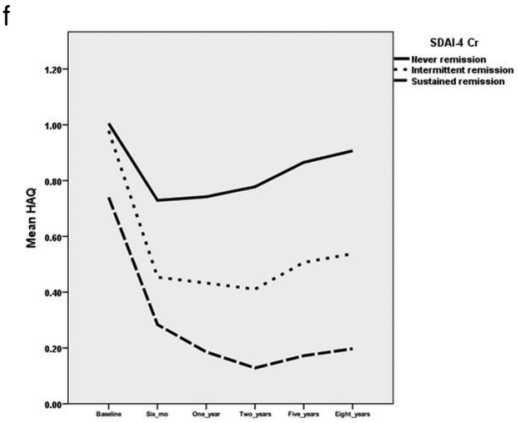

Figure 2 (A-F) Mean Health Assessment Questionnaire from baseline to 6 months, 1, 2, 5 and 8 years in patients in sustained remission, intermittent remission or never in remission by DAS28 $\mathrm{Cr}(\mathrm{A})$, DAS28-3 $\mathrm{Cr}$ (B), Boolean $\mathrm{Cr}(\mathrm{C})$, Boolean-3 $\mathrm{Cr}(\mathrm{D})$, SDAI $\mathrm{Cr}(\mathrm{E})$, SDAl-4 $\mathrm{Cr}(\mathrm{F})$. The differences between remission groups at baseline were significant for all criteria, overall $p=0.013$ (a), 0.018 (b), 0.001 (c), 0.048 (d), 0.009 (e) and 0.044 (f). At all follow-up visits, the overall $p$ value for the differences between remission groups was $<0.001$ by all criteria (Kruskal-Wallis test).

Boolean-3 Cr $7 \%$ vs $13 \%$, $\mathrm{p}=0.001$; by the SDAI Cr $4 \%$ vs $7 \%, \mathrm{p}=0.001$; and by the SDAI-4 Cr $7 \%$ vs $11 \%, \mathrm{p}=0.001$.

\section{DISCUSSION}

Long-term sustained remission in RA is expected to be associated with a favourable outcome as regards disability and joint destruction. However, this may not be readily demonstrated since the validity of available remission criteria in long-term observational studies is still insufficiently known. Therefore, we have, in our longterm observational study of patients with early RA, used the DAS28 remission criterion (DAS28 Cr) as well as the recently proposed ACR/EULAR remission criteria-the Boolean variant-and the SDAI Cr. In addition, these criteria have also been modified by excluding the PatGA.

In the present study, remission by the DAS28 Cr was about twice as frequent as that by the Boolean Cr. The frequencies found were similar to those in other studies. Thus, in one inception cohort, $33.7 \%$ of the patients had, 1 year after enrolment, achieved remission by the DAS28 Cr, $13.8 \%$ by the Boolean $\mathrm{Cr}$ and $16.8 \%$ by the SDAI Cr. ${ }^{15}$ Furthermore, in unselected patients with established RA, 28\% were found to be in DAS28 Cr remission while only $7 \%$ had achieved remission by the Boolean Cr. ${ }^{16}$ Cross-sectional data from two large registries of patients with established RA showed that only $5-6.2 \%$ of the patients had achieved remission by the Boolean $\mathrm{Cr}$ and $6.9-10.1 \%$ by the SDAI Cr. ${ }^{17}$

In this study, long-term sustained remission was considerably less frequent, with $3 \%$ of the patients by the Boolean Cr, $5 \%$ by the SDAI Cr and $14 \%$ by the DAS28 Cr. In the study by Shahouri et $a l^{17}$, the probability of having two or more visits in remission during 2 years was at most 2.8\% for the Boolean Cr and 4.2\% for the SDAI. In the ERAS study, remission by the DAS criterion $\leq 1.6$ was achieved by $11 \%$ of the patients at all three follow-up visits at 3,4 and 5 years, only slightly less than the $14 \%$ found in our study. ${ }^{18}$ A study on patients with established RA treated in clinical practice reports that only a minority of those who achieved remission remained in remission during follow-up, irrespective of the criteria used..$^{19}$ In an editorial to that article, Aletaha ${ }^{20}$ stresses the importance of considering sustained remission as 'an outcome measure of successful treatment', which is in line with the purpose of this study.

Remission criteria should satisfy the requirements of absent or minimal disease activity and no or little future disability or joint damage. However, if the criteria are too stringent, overtreatment may follow. Conversely, if too permissive criteria are chosen, patients with significant disease activity may be classified as being in remission and thus miss adequate treatment.

Sometimes, it may be difficult to decide whether tenderness or swelling is related to the disease activity or not. During the long-term course of RA, tender or 
swollen joints may be unrelated to disease activity and lead to misclassification. Thus, as has also been pointed out by Thiele et $a l^{16}{ }^{16}$ tender and swollen joints may reflect some other coexisting rheumatic disorder or represent sequels of RA. Tenderness in non-swollen joints may also be due to painful disorders like fibromyalgia, which occurs in 12-20\% of patients with RA. ${ }^{21}$ All this may cause misinterpretations and missed remissions, conceivably more often by the more stringent criteria.

The very low cut-off required for VAS PatGA $(\leq 1 \mathrm{~cm})$ has been found to be a limiting factor for achieving remission by the Boolean $\mathrm{Cr}^{22}$ In most remission criteria, a VAS PatGA is included that represents the patient's perspective. However, its disease specificity may be questioned since an elevated PatGA may be due to, for example, low back pain or other comorbidities. ${ }^{16}$ In agreement with others, we found that VAS PatGA correlated well with VAS pain $(\mathrm{r}=0.80,0.81,0.86$ and 0.83 at the follow-up visits at $1,2,5$ and 8 years), a common symptom not only of current disease activity but also of various comorbidities. ${ }^{23} 24$ In the present study, the phrasing (translated to English) was similar to that used by others: 'How do you feel to-day with reference to your rheumatic disease?' The possibility that the questions, although seemingly clear, may cause misclassifications is supported by Thiele $e t a l^{16}$ who report that $91 \%$ of the patients in their study gave the same $(77 \%)$ or almost the same (14\%) rating to the questions 'describe your health to-day' and 'assess the activity of your disease'. A way to overcome this bias would be to phrase the questions more distinctly.

The low frequency of sustained remission by the Boolean Cr suggests that they may be too stringent to be suitable for long-term studies in clinical practice. In the present study, the exclusion of PatGA from the Boolean $\mathrm{Cr}$ and the SDAI Cr resulted in an increase in the rate of sustained remission from $3 \%$ to $9 \%$ and $5 \%$ to $8 \%$, respectively. This effect was not seen with the DAS28 Cr. This may suggest that missed remissions due to non-RA-related high VAS PatGA are less common when the DAS28 Cr are used. This could partly be explained by the fact that the contribution of PatGA to DAS28 is only $15 \% .^{25}$ A VAS PatGA of $2-3 \mathrm{~cm}$ was not infrequent in patients in sustained remission by DAS28Cr (data not shown).

The formula for DAS28 allocates twice as much weight to the number of tender joints as to the number of swollen joints. This, for example, means that while remission can be missed with one swollen joint and three tender joints, it can be achieved with one tender joint and five or even more swollen joints. ${ }^{25}$ Consequently, the DAS28 Cr has been criticised for allowing classification of patients as being in remission in spite of having several swollen joints, not compatible with a state of remission. ${ }^{6}$ However, in the group of patients who achieved sustained remission in the present study, only a minority had more than a few tender or swollen joints. So, it seems reasonable to use DAS28 Cr for the definition of sustained remission in this patient material. Furthermore, tender joints in the absence of swelling have been shown to be unrelated to active synovitis diagnosed by ultrasound or power Doppler, which suggests that tender joints, which are not swollen, may be a source of misclassification. ${ }^{26}$

Gender may influence remission rates. ${ }^{16}$ In the BARFOT study, we have earlier reported that sustained remission from years $2-5$, determined by the DAS28 $<2.6$ criterion, was significantly less frequent in women than in men. ${ }^{27}$ Furthermore, women had higher DAS28 after 5 and 8 years than men, but no gender difference was observed in radiographic progression. ${ }^{28}{ }^{29}$ Similarly, in the present study, sustained remission by all criteria was significantly less frequent in women than in men, while radiographic joint damage was similar (data not shown). The explanation to this inconsistency is probably that non-inflammatory pain confounds the measurement of disease activity in women.

Ideally, a state of sustained remission should indicate that disease activity is virtually absent and thus eliminate or minimise the risk of further joint damage. Even if no study has shown a complete arrest of joint damage over extended periods of time, two studies lend support that this may be possible. In the Fin-RaCo study, sustained remission by the DAS28 $\mathrm{Cr}$ over 2 years was associated with only modest radiographic joint damage, and in the PREMIER study, sustained remission during the second year was associated with arrest of joint damage, irrespective of the therapy given. ${ }^{30} 31$ As a consequence, the authors proposed that sustained remission should be the ultimate goal of treatment of RA.

The validity of the Boolean $\mathrm{Cr}$ and SDAI Cr has been established in short-term or medium-term studies by demonstrating satisfactory likelihood ratios for 'good radiographic outcome' of being in remission. ${ }^{6}{ }^{13}$ In this study, as in another long-term study, ${ }^{18}$ radiographic progression was also common in patients in sustained remission, regardless of the criteria used, and consequently the likelihood ratios were small. However, the degree of radiographic damage was minor in the groups of patients in sustained remission and often below 'the lowest value of minor radiological change'. ${ }^{12}$ Similar results were obtained using different cut-off values for radiographic progression (data not shown). So, sustained remission by all criteria seems to be associated with slow long-term radiographic progression.

The progression of radiographic joint damage in patients in sustained remission may be explained by flares of disease activity between the four assessment points. Here we have the main limitation of this study, that is, the scarcity of follow-up visits during the 8-year follow-up. However, at the time this study was planned, it was not feasible to predetermine controls tight enough to be able to catch up flares. Another possible explanation is the presence of subclinical inflammation, which may cause progressive joint damage in patients without clinical evidence of inflammation. ${ }^{32}$ However, in the 
patients in sustained remission in this study, the radiographic joint damage over the 8 years was very limited and similar between criteria. Furthermore, a state of sustained remission by all criteria was associated with lasting return to a functional level, corresponding to that of an age-matched and sex-matched general population. ${ }^{33}$

\section{CONCLUSION}

The present study has focused on finding suitable criteria for identifying patients in long-term sustained remission to be used in the assessment of the disease course and outcome of RA. The data suggest that the Boolean Cr, although performing well in clinical trials, are very stringent and bring about risks for misclassifications mainly due to the requirement of a very low PatGA and hence risk for over treatment. Similar objections may be made to the use of the SDAI Cr, which may make these criteria less appropriate for use in long-term studies in which PatGA frequently may be increased due to comorbidities. Both these sets of criteria classified more patients in sustained remission when PatGA was excluded. However, using criteria without PatGA means that the much wanted patient perspective of the criteria is abandoned. ${ }^{34}$ Furthermore, cut-offs for remissions have not been established for criteria without VAS PatGA. The DAS28 Cr performed reasonably well in this 8-year study and presented very little of previously observed drawbacks. In spite of more patients in sustained remission by DAS28 Cr, radiographic damage and disability were apparently not worse than what was seen with the other criteria and the patients' perspective was preserved. The DAS28 Cr may therefore still be used in long-term observational studies until more accurate criteria are available.

Acknowledgements The authors would like to thank the following members of the BARFOT Study Group for valuable cooperation: Sofia Ajeganova, Stefan Bergman, Åsa Häggström, Catharina Keller, Ido Leden, Bengt Lindell,

Christoffer Schaufelberger, Maria Söderlin, Annika Teleman, Jan Theander and Anneli Östenson. They also thank Dr Kristina Albertsson for participating in the scoring of the radiographs.

Contributors BS inspired the study, designed it, analysed the data, performed the statistics and led the writing of the manuscript. MLEA was responsible for sampling and data recovery. S-VB participated in the data analysis and discussion. KF was responsible for the radiographic assessments including the scoring of radiographs. IH participated in the design and interpretation of data as well as manuscript preparation. All authors have critically revised and approved the final version of the manuscript.

Funding This work was supported by grants from the Swedish Rheumatism Association, King Gustaf V 80-year Fund, the Thelma Zoégas foundation in Helsingborg, and Stiftelsen för Rörelsehindrade i Skåne.

Competing interests None.

Patient consent Obtained.

Ethics approval All patients gave their informed consent and the Ethics committees approved the study which was performed in accordance with the Declaration of Helsinki.

Provenance and peer review Not commissioned; externally peer reviewed. Data sharing statement No additional data are available.
Open Access This is an Open Access article distributed in accordance with the Creative Commons Attribution Non Commercial (CC BY-NC 3.0) license, which permits others to distribute, remix, adapt, build upon this work noncommercially, and license their derivative works on different terms, provided the original work is properly cited and the use is non-commercial. See: http:// creativecommons.org/licenses/by-nc/3.0/

\section{REFERENCES}

1. Smolen JS, Aletaha D, Bijlsma JW, et al. Treating rheumatoid arthritis to target: recommendations of an international task force. Ann Rheum Dis 2010;69:631-7.

2. Pinals RS, Masi AT, Larsen RA. Preliminary criteria for clinical remission in rheumatoid arthritis. Arthritis Rheum 1981;24:1308-15.

3. van der Heijde DM, van't Hof M, van Riel PL, et al. Development of a disease activity score based on judgment in clinical practice by rheumatologists. J Rheumatol 1993;20:579-81.

4. Prevoo ML, van Gestel AM, van't Hof, MA et al. Remission in a prospective study of patients with rheumatoid arthritis. American Rheumatism Association preliminary remission criteria in relation to the disease activity score. Br J Rheumatol 1996;35:1101-5.

5. Smolen JS, Breedveld FC, Schiff MH, et al. A simplified disease activity index for rheumatoid arthritis for use in clinical practice. Rheumatology (Oxford) 2003;42:244-57.

6. Felson DT, Smolen JS, Wells G, et al. American College of Rheumatology/European League against Rheumatism provisional definition of remission in rheumatoid arthritis for clinical trials. Ann Rheum Dis 2011;70:404-13.

7. Svensson B, Schaufelberger C, Teleman A, et al. Remission and response to early treatment of $R A$ assessed by the disease activity score. BARFOT study group. Better anti-rheumatic farmacotherapy. Rheumatology (Oxford) 2000;39:1031-6.

8. Ekdahl C, Eberhardt $\mathrm{K}$, Andersson SI, et al. Assessing disability in patients with rheumatoid arthritis. Use of a Swedish version of the Stanford Health Assessment Questionnaire. Scand J Rheumatol 1988;17:263-71.

9. Prevoo ML, van't Hof MA, Kuper $\mathrm{HH}$, et al. Modified disease activity scores that include twenty-eight-joint counts. Development and validation in a prospective longitudinal study of patients with rheumatoid arthritis. Arthritis Rheum 1995;38:44-8.

10. Fransen J, Creemers MC, Van Riel PL. Remission in rheumatoid arthritis: agreement of the disease activity score (DAS28) with the ARA preliminary remission criteria. Rheumatology (Oxford) 2004;43:1252-5.

11. van der Heijde D. How to read radiographs according to the Sharp/ van der Heijde method. J Rheumatol 2000;27:261-3.

12. van der Heijde $D$, Landewe $R$, van Vollenhoven $R$, et al. Level of radiographic damage and radiographic progression are determinants of physical function: a longitudinal analysis of the TEMPO trial. Ann Rheum Dis 2008;67:1267-70.

13. Lillegraven S, Prince FH, Shadick NA, et al. Remission and radiographic outcome in rheumatoid arthritis: application of the 2011 ACR/EULAR remission criteria in an observational cohort. Ann Rheum Dis 2012;71:681-6.

14. Bruce B, Fries JF. The Stanford Health Assessment Questionnaire: dimensions and practical applications. Health Qual Life Outcomes 2003;1:20.

15. Sakellariou G, Scire CA, Verstappen SM, et al. In patients with early rheumatoid arthritis, the new ACR/EULAR definition of remission identifies patients with persistent absence of functional disability and suppression of ultrasonographic synovitis. Ann Rheum Dis 2013;72:245-9.

16. Thiele K, Huscher D, Bischoff S, et al. Performance of the 2011 ACR/EULAR preliminary remission criteria compared with DAS28 remission in unselected patients with rheumatoid arthritis. Ann Rheum Dis 2013;72:1194-9.

17. Shahouri SH, Michaud K, Mikuls TR, et al. Remission of rheumatoid arthritis in clinical practice: application of the American College of Rheumatology/European League Against Rheumatism 2011 remission criteria. Arthritis Rheum 2011;63:3204-15.

18. Jayakumar K, Norton S, Dixey J, et al. Sustained clinical remission in rheumatoid arthritis: prevalence and prognostic factors in an inception cohort of patients treated with conventional DMARDS. Rheumatology (Oxford) 2012;51:169-75.

19. Prince FH, Bykerk VP, Shadick NA, et al. Sustained rheumatoid arthritis remission is uncommon in clinical practice. Arthritis Res Ther 2012;14:R68.

20. Aletaha D. Nothing lasts forever-a critical look at sustained remission. Arthritis Res Ther 2012;14:116. 
21. Yunus MB. The prevalence of fibromyalgia in other chronic pain conditions. Pain Res Treat 2012;2012:584573.

22. Studenic $P$, Smolen JS, Aletaha D. Near misses of ACR/EULAR criteria for remission: effects of patient global assessment in Boolean and index-based definitions. Ann Rheum Dis 2012;71:1702-5.

23. Masri KR, Shaver TS, Shahouri SH, et al. Validity and reliability problems with patient global as a component of the ACR/EULAR remission criteria as used in clinical practice. $J$ Rheumatol 2012;39:1139-45.

24. Studenic P, Radner H, Smolen JS, et al. Discrepancies between patients and physicians in their perceptions of rheumatoid arthritis disease activity. Arthritis Rheum 2012;64:2814-23.

25. Belmonte Serrano MA. Is the DAS28 score the most adequate method to estimate activity in Rheumatoid Arthritis? Clinimetric considerations and simulations scenarios. Reumatol Clin 2008;4:183-90.

26. Rees JD, Pilcher J, Heron C, et al. A comparison of clinical vs ultrasound determined synovitis in rheumatoid arthritis utilizing gray-scale, power Doppler and the intravenous microbubble contrast agent 'Sono-Vue'. Rheumatology (Oxford) 2007:46:454-9.

27. Forslind K, Hafstrom I, Ahlmen M, et al. Sex: a major predictor of remission in early rheumatoid arthritis? Ann Rheum Dis 2007;66:46-52.

28. Hafstrom I, Bala V, Albertsson K, et al. Joint destruction in early rheumatoid arthritis over 8 years is similar in women and men despite apparently higher disease activity and poorer function in women. Ann Rheum Dis 2011;70:709-10.

29. Ahlmén M, Svensson B, Albertsson K, et al. Influence of gender on assessments of disease activity and function in early rheumatoid arthritis in relation to radiographic joint damage. Ann Rheum Dis 2010;69:230-3.

30. Makinen $\mathrm{H}$, Kautiainen $\mathrm{H}$, Hannonen $\mathrm{P}$, et al. Sustained remission and reduced radiographic progression with combination disease modifying antirheumatic drugs in early rheumatoid arthritis. $J$ Rheumatol 2007;34:316-21.

31. Aletaha D, Funovits J, Breedveld FC, et al. Rheumatoid arthritis joint progression in sustained remission is determined by disease activity levels preceding the period of radiographic assessment. Arthritis Rheum 2009;60:1242-9.

32. Brown AK, Conaghan PG, Karim Z, et al. An explanation for the apparent dissociation between clinical remission and continued structural deterioration in rheumatoid arthritis. Arthritis Rheum 2008;58:2958-67.

33. Krishnan E, Sokka T, Hakkinen A, et al. Normative values for the Health Assessment Questionnaire disability index: benchmarking disability in the general population. Arthritis Rheum 2004;50: 953-60

34. Khan NA, Spencer HJ, Abda E, et al. Determinants of discordance in patients' and physicians' rating of rheumatoid arthritis disease activity. Arthritis Care Res 2012;64:206-14. 